\title{
Frequency and Pathogenesis of Silent Subcortical Brain Infarction in Acute First-ever Ischemic Stroke
}

\author{
Tomohide Adachi*, Shotai Kobayashi and Shuhei Yamaguchi
}

\begin{abstract}
Objectives We have often observed silent subcortical brain lesions on CT or MRI in first-ever ischemic stroke, but there is little published information on the relationship of these lesions to stroke subtypes. Here, we describe the incidence of MRI-detected silent subcortical brain lesions, including infarctions and white matter lesions, in a series of patients with first-ever ischemic stroke classified according to stroke subtypes. We also discuss the pathogenesis of these silent subcortical lesions.

Patients We evaluated 171 patients with acute first-ever ischemic stroke.

Methods The subjects were divided into three groups: lacunar, atherothrombotic and cardioembolic infarction groups. We evaluated silent subcortical brain infarction (SSBI), enlargement of perivascular space (EPS), and other white-matter lesions using MRI.

Results Hypertension was observed in $67.6 \%$ of lacunar infarction, $57.1 \%$ of atherosclerotic infarction, and $54.1 \%$ of cardioembolic infarction. SSBI was more frequently observed in lacunar infarction than the others (lacunar vs. atherothrombotic vs. cardiogenic infarction, $81.5 \%$ vs. $44.4 \%$ vs. $42.1 \%$, $p=0.006)$. High-grade EPS (grade 2 or higher) was also observed more frequently in lacunar infarction than in the others (lacunar vs. atherothrombotic vs. cardiogenic infarction, $63.3 \%$ vs. $24.2 \%$ vs. $0 \%, \mathbf{p}<0.001)$. Scheltens' score of silent subcortical lesions was significantly higher in lacunar infarction than in the others.

Conclusions The frequency of silent subcortical ischemic brain lesions was significantly higher in lacunar infarction than in atherosclerotic or cardioembolic infarction. We suggest that the pathogenesis of silent subcortical ischemic brain lesions is common to that of lacunar infarction, that is, small-vessel vasculopathy.
\end{abstract}

(Internal Medicine 41: 103-108, 2002)

Key words: acute ischemic stroke, silent infarct, magnetic resonance imaging, small-vessel disease

\section{Introduction}

We have often observed silent subcortical brain lesions on brain CT or MRI in the elderly. Several previous studies have reported the incidence of silent brain infarction, i.e. infarction not associated with any apparent symptoms. In an apparently healthy population, the frequency of silent brain infarction was $7.9 \%-47 \%(1-8)$. In ischemic stroke patients, the frequency was reported to be $11 \%-68 \%$ on the basis of CT and MRI findings $(4,7,9-11)$. We have previously reported that $10.6 \%-$ $13 \%$ of apparently healthy adults had silent subcortical brain infarction as revealed by MRI $(1,5)$. Shinkawa et al found that $12.9 \%$ of autopsy cases had silent cerebral infarction in the Hisayama study (6). Okada et al reported that $89 \%$ of patients with initial hypertensive brain hemorrhage had silent lacunes manifested on MRI (12). They indicated that hypertensive brain hemorrhage exhibits pathological changes similar to those associated with lacunar brain infarction. However, few reports have described the frequency of silent brain infarction in firstever ischemic stroke classified according to the subtypes of ischemic stroke, such as lacunar infarction, atherothrombotic infarction, and cardioembolic infarction $(10,11)$, with only one report being based on MRI findings (11). There has been no report of the frequency of silent lesions among stroke subtypes in Japanese. The pathogenesis of silent brain infarction is not obvious, and the frequencies of other subcortical lesions, such as periventricular hyperintensity, white-matter hyperintensity, and enlargement of perivascular space, among the subtypes of ischemic stroke are also unclear. The relation between the subtypes of ischemic stroke and the pathogenesis of these silent subcortical lesions has not yet been clarified. In the present study, we investigated the frequency of MRI-detected silent subcortical brain infarction (SSBI) and other silent brain lesions in patients with first-ever ischemic stroke according to subtype. The results provide a clue to the possible pathogenesis of silent subcortical brain lesions.

\section{Patients and Methods}

We evaluated 171 patients (103 men and 68 women; ages

From the Department of Internal Medicine III, Shimane Medical University, Izumo, *Present address: Department of Neurology, Isuzu Hospital, Tokyo Received for publication March 21, 2001; Accepted for publication October 26, 2001

Reprint request should be addressed to Dr. Tomohide Adachi, the Department of Neurology, Isuzu Hospital, 6-21-10 Minami-Ooi, Shinagawa-ku, Tokyo 1400013 
ranging from 26 to 93 years; mean age 69 years) with acute first-ever ischemic stroke who had been admitted sequentially to Shimane Medical University Hospital from 1994 to 1997. The information regarding the subjects was gathered by means of a retrospective review of the patients' medical records. These subjects were divided into three groups: atherothrombotic infarction (53 patients, mean age 68 years), lacunar infarction (74 patients, mean age 67 years), and cardioembolic infarction (37 patients, mean age 73 years). Seven cases were unclassified and thus were excluded from this study. This classification followed the criteria of NINDS 90 (13). We used information regarding the onset of stroke, neurological findings, risk factors of ischemic stroke, findings of brain MRI, and intraand extracranial vascular lesions seen on MR angiography, conventional angiography and duplex carotid sonography, as well as the results of electrocardiography, and ultrasound echocardiography. Atherothrombotic infarction was defined as cases with severe stenosis $(>50 \%)$ or occlusion of the intracranial or extracranial large vessel on the affected side. Such patients showed cortical symptoms and/or cortical infarction or large subcortical infarction. Lacunar infarction was defined as small subcortical infarction less than $2 \mathrm{~cm}$ in diameter, without cortical symptoms and large vessel lesions. Cardioembolic infarction was diagnosed in patients who showed sudden-onset neurological deficit with arrhythmia, e.g., atrial fibrillation, cardiomyopathy, or an intracardiac embolic source on an ultrasound echocardiogram. Lacunar-sized small infarction resulting from large vessel disease was classified as atherothrombotic infarction, and that resulting from cardioembolism was classified as cardioembolic infarction, following the pathogenesis in each case.

We assessed the risk factors for ischemic stroke, i.e., age, sex, hypertension, diabetes mellitus, hypercholesterolemia (history or total cholesterol greater than $220 \mathrm{mg} / \mathrm{dl}$ at admission), current smoking and atrial fibrillation. Laboratory analysis involved complete blood cell count and blood coagulation including fibrinogen, prothrombin time, active partial thromboplastin time, and biochemistry studies including fasting blood glucose, total cholesterol, triglyceride, and high-density lipoprotein cholesterol.

Brain MRI was performed using a 1.5T superconducting unit (Signa Advantage, GE; Gyroscan ACS-NT, Philips). We obtained T2-weighted images (TR: 3,800-2,000 msec, TE: 140-100 msec), T1-weighted images (TR: 400-350 msec, TE: 14-12 $\mathrm{msec}$ ), and proton density-weighted images (TR: 3,800 3,500 msec, TE: $26-20 \mathrm{msec}$ ). Transaxial images were obtained with $6 \mathrm{~mm}$ thickness without a gap. Two-dimensional Fourier transformation of the images and a $256 \times 256$ data acquisition matrix were used. We determined the high signal intensity area responsible for neurological symptoms from the T2-weighted images.

We defined SSBI as high intensity lesion having a size of $\geq 3 \mathrm{~mm}$ on T2-weighted images and low intensity on T1weighted images, following the criteria of the Workshop Study on "Diagnostic Criteria for Asymptomatic Cerebrovascular Diseases" (14). We measured the size of the high-intensity le- sion using axial views. Enlargement of perivascular space (EPS) was defined as a lesion with a maximum diameter of $<3 \mathrm{~mm}$, which was seen as a spotty high signal intensity on the T2weighted image and the same intensity as cerebrospinal fluid on the proton density-weighted image $(14,15)$. EPS was classified into 4 grades, based on lesions at the basal ganglia: Grade 0: no lesion, Grade 1: 1-5 lesions, Grade 2: 6-10 lesions, Grade 3: $>10$ lesions. We defined lesions of grade 2 or higher as highgrade EPS. We semiquantitatively evaluated silent subcortical lesions, including SSBI and EPS, on the T2-weighted images, as well as periventricular hyperintensity $(\mathrm{PVH})$, white matter hyperintensity (WMH), basal ganglia hyperintensity (BGH), and infratentorial hyperintensity (ITH), using the modified method reported by Scheltens et al $(16,27)$. High intensity lesions were scored as either five or six points according to the number and diameter of lesions in each area, such as the frontal, parietal, temporal, and occipital regions. The total score was calculated using these values.

Three observers evaluated the MRIs in a blind fashion. One observer evaluated all the MRIs, while the two other observers evaluated 25 MRIs independently, in order to estimate the reliability of the interpretation of the radiological findings. These observers were unaware of the nature of this study, and had no clinical information regarding the patients, except for that shown on the MRIs.

Lesions in the extracranial carotid artery were evaluated using conventional angiography, and/or carotid duplex sonography (DCS) by means of a $7.5 \mathrm{MHz}$ probe (Ultramark 9 , ATL; SSD-2000, Aloka). We classified vascular lesions caused by atherosclerosis into categories of mild stenosis $(<50 \%)$, severe stenosis $(\geq 50 \%)$, and occlusion at the origin of internal carotid artery (ICA). We referred to the example of the European Carotid Surgery Trial and determined the ratio of the diameter of the internal lumen to the whole vessel on angiography and DCS (17). The intracranial arteries were evaluated by 3D-time-of-flight MR angiography and/or conventional cerebral angiography. We defined mild stenosis as a partial narrowing of the caliber of the artery, severe stenosis as partial disappearance of the flow signal, and occlusion as complete interruption of the flow signal $(18,19)$.

The nominal variables (risk factors for ischemic stroke, frequency of SSBI and grades of EPS) were analyzed using the chi-square test. The relation between age and stroke subtypes was analyzed using the analysis of variance. Differences of PVH, WMH, BGH, ITH, and total scores among stroke subtypes were analyzed using the Kruskal-Wallis test. The relation between SSBI and cerebrovascular risk factors was analyzed using uni- and multivariate logistic regressions. All variables with $\mathrm{p}<0.10$ in the univariate analysis were included in multivariate analysis. A value of $p<0.05$ was used as the criterion of statistical significance. The inter-observer reliability in scoring the silent brain lesions was assessed using Pearson's correlation coefficient. 


\section{Results}

Inter-observer reliability was good for all scores relating to silent brain lesions ( $R=0.763$ to 0.895 , average 0.845 ).

The frequencies of risk factors for ischemic stroke are shown in Table 1. There was no significant difference in age among the three groups. Hypertension was more frequent in the lacunar infarction group, but no statistically significant difference was seen among the three groups; lacunar infarction vs. atherothrombotic infarction vs. cardioembolic infarction was $67.6 \%$ vs. $60.4 \%$ vs. $56.8 \%$. Atrial fibrillation was significantly more frequent in the cardioembolic infarction group (56.8\%) than in the others. Laboratory values showed no significant differences among the three groups.

In the atherothrombotic infarction group, $34.3 \%$ of the subjects showed severe stenosis or occlusion of the homolateral internal carotid artery; severe stenosis and occlusion were seen in $25 \%$ and $75 \%$, while $57.1 \%$ of subjects had stenosis or occlusion of the homolateral middle cerebral artery of the affected side (severe stenosis and occlusion in $20 \%$ and $80 \%$, respec- tively). Other patients with atherothrombotic infarction showed infratentorial infarction and stenosis of the anterior cerebral artery. There was no case with large vessel lesions in the lacunar infarction group. In the cardioembolic infarction group, the frequency of occlusive lesion of the homolateral internal carotid artery was $9.5 \%$, while the frequency of occlusion of the middle cerebral artery was $19.0 \%$.

The frequency of SSBI was $56.7 \%$ in all groups (Table 2). The incidence of SSBI was higher in the lacunar infarction group than in the other two groups (lacunar infarction vs. atherothrombotic infarction vs. cardioembolic infarction, $81.1 \%$ vs. $45.3 \%$ vs. $43.2 \%, \mathrm{p}=0.006$ ). The frequency of high-grade EPS (grade 2 or higher) was $31.6 \%$ all groups. The grade of EPS was also higher in the lacunar infarction group than in the other two groups $(\mathrm{p}<0.001)$. High-grade EPS amounted to $63.5 \%$ in the lacunar infarction group, $24.5 \%$ in the atherothrombotic infarction group, and $0 \%$ in the cardioembolic infarction group.

Scheltens' score for PVH, BGH, and the total score of silent subcortical lesions were significantly higher in lacunar infarction than in the others (PVH, BGH, and total score; $p=0.001$,

Table 1. Frequencies of Risk Factors and Atherosclerotic Complications Among Subtypes of Cerebral Infarction

\begin{tabular}{lcccc}
\hline Risk factor & $\begin{array}{c}\text { Atherothrombotic } \\
\text { infarction } \\
(\mathrm{n}=53)\end{array}$ & $\begin{array}{c}\text { Lacunar } \\
\text { infarction } \\
(\mathrm{n}=74)\end{array}$ & $\begin{array}{c}\text { Cardioembolic } \\
\text { infarction } \\
(\mathrm{n}=37)\end{array}$ & P-value \\
\hline Age & $71.6 \pm 10.3$ & $71.2 \pm 9.3$ & $73.8 \pm 10.0$ & 0.417 \\
Sex (male), (\%) & $36(67.9)$ & $50(67.6)$ & $23(62.1)$ & 0.774 \\
Hypertension, $(\%)$ & $32(60.4)$ & $50(67.6)$ & $20(54.1)$ & 0.441 \\
Diabetes mellitus, $(\%)$ & $21(39.6)$ & $22(29.7)$ & $7(18.9)$ & 0.120 \\
Hyperlipidemia, $(\%)$ & $6(11.3)$ & $16(21.6)$ & $3(8.1)$ & 0.124 \\
Atrial fibrillation, $(\%)$ & $1(1.9)$ & $2(2.7)$ & $21(56.8)$ & $<0.001$ \\
Current smoking, $(\%)$ & $15(28.3)$ & $26(35.1)$ & $6(16.2)$ & 0.447 \\
Ischemic heart disease, $(\%)$ & $5(9.4)$ & $10(13.5)$ & $5(13.5)$ & 0.687 \\
ASO, $(\%)$ & $2(3.1)$ & $2(2.7)$ & $2(5.4)$ & 0.948 \\
\hline
\end{tabular}

ASO: arteriosclerosis obliterans.

Table 2. Frequency of Silent Subcortical Lesions

\begin{tabular}{lccccc}
\hline $\begin{array}{c}\text { Silent } \\
\text { subcortical } \\
\text { lesions }\end{array}$ & $\begin{array}{c}\text { Total } \\
(\mathrm{n}=171)\end{array}$ & $\begin{array}{c}\text { Atherothrombotic } \\
\text { infarction } \\
(\mathrm{n}=53)\end{array}$ & $\begin{array}{c}\text { Lacunar } \\
\text { infarction } \\
(\mathrm{n}=74)\end{array}$ & $\begin{array}{c}\text { Cardioembolic } \\
\text { infarction } \\
(\mathrm{n}=37)\end{array}$ & P-value \\
\hline SSBI, (\%) & $97(56.7)$ & $24(45.3)$ & $60(81.1)$ & $16(43.2)$ & 0.006 \\
EPS, (\%) & $54(31.6)$ & $13(24.5)$ & $47(63.5)$ & $0(0.0)$ & $<0.001$ \\
PVH score & 5.4 & 5.0 & 7.6 & 2.9 & 0.001 \\
WMH score & 10.9 & 10.4 & 14.6 & 6.6 & $<0.001$ \\
BGH score & 11.3 & 8.1 & 19.7 & 4.8 & $<0.001$ \\
Total score & 28.8 & 24.6 & 43.7 & 15.1 & $<0.001$ \\
\hline
\end{tabular}

*Grade 2 or greater enlargement of perivascular space. SSBI: silent subcortical brain infarction, EPS: enlargement of perivascular space. Score is average of Scheltens' score of silent subcortical lesions. 
ADACHI et al

Table 3. Univariate Analysis of Risk Factors for SSBI

\begin{tabular}{lcc}
\hline & Odds ratio (95\% confidence interval) & P-value \\
\hline Age & $1.01(0.99-1.04)$ & 0.323 \\
Sex, female & $0.40(0.19-0.83)$ & 0.015 \\
Hypertension & $2.89(1.50-5.56)$ & 0.001 \\
Diabetes mellitus & $1.86(0.89-3.88)$ & 0.095 \\
Hyperlipidemia & $1.12(0.43-2.92)$ & 0.809 \\
Atrial fibrillation & $0.60(0.27-1.34)$ & 0.218 \\
Current smoking & $1.18(0.59-2.33)$ & 0.639 \\
Alcohol consumption & $2.37(1.21-4.62)$ & 0.011 \\
\hline
\end{tabular}

Table 4. Multivariate Analysis of Risk Factors for SSBI

\begin{tabular}{lcccc}
\hline & \multicolumn{4}{c}{ Odds ratio } \\
\cline { 2 - 5 } & $\begin{array}{c}\text { Whole } \\
\text { infarction }\end{array}$ & $\begin{array}{c}\text { Atherothrombotic } \\
\text { infarction }\end{array}$ & $\begin{array}{c}\text { Lacunar } \\
\text { infarction }\end{array}$ & $\begin{array}{c}\text { Cardioembolic } \\
\text { infarction }\end{array}$ \\
\hline Age & - & 1.18 & - & - \\
Sex, female & 0.46 & - & 0.15 & - \\
Hypertension & $2.97^{*}$ & $12.03^{*}$ & $13.91^{*}$ & 0.27 \\
Diabetes mellitus & 1.96 & - & - & 3.05 \\
Current smoking & - & 0.56 & - & - \\
Alcohol consumption & 1.63 & - & - & 6.85 \\
\hline
\end{tabular}

$* \mathrm{p}<0.05$. Blanks indicate data not applicable.

$<0.001$, and $<0.001)$. WMH score was significantly higher in the lacunar infarction group than in the other two groups $(\mathrm{p}<0.001)$ (Table 2).

The results of univariate analysis of the cerebrovascular risk factors for SSBI are shown in Table 3. Hypertension and alcohol consumption were significant predictors for SSBI. Hypertension, sex, diabetes mellitus, and alcohol consumption were entered into the multivariate analysis (Table 4). Hypertension was the only significant independent predictor for SSBI. Similar univariate analyses for SSBI in the lacunar infarction group, atherothrombotic infarction group, and cardioembolic infarction group were performed (data not shown). Only hypertension was a significant predictor for SSBI in the lacunar infarction and atherothrombotic infarction groups.

\section{Discussion}

We present here the frequencies of SSBI according to the subtypes of first-ever ischemic stroke as revealed by MRI. The frequency of SSBI was higher in the lacunar infarction group than in the atherothrombotic infarction and cardioembolic infarction groups. In previous reports, the frequency of SSBI in lacunar infarction was also higher than that in other categories of infarction $(10,12)$. Boon et al reported that the incidence of silent small infarcts was $29.6 \%$ in small deep infarctions examined by CT (10). Chamorro et al also reported that the inci- dence of silent infarction was $41 \%$ in lacunar infarction examined by MRI (11). Lacunar infarction resulted mainly from lipohyalinosis of a small penetrating artery (20-22). We have reported that persons with SSBI should be considered at high risk for clinical subcortical brain infarction or brain hemorrhage, and indicated that the most important mechanism for SSBI was hypertensive small-vessel vasculopathy (5). Laloux et al reported that in patients with silent cerebral infarcts, smallvessel disease may in most cases be the cause of their recent symptomatic cerebral ischemia (23). Boon et al also suggested that small-vessel vasculopathy was a common underlying mechanism of generation of silent small deep subcortical lesions and first symptomatic small deep infarct (10). These reports and the present study suggest that the pathogenesis of SSBI is common to that of lacunar infarction. We suggest that SSBI and lacunar infarction are caused mainly by arteriosclerosis of small penetrating arteries. In our patients, the frequency of SSBI was higher than that described in previous reports. This might reflect a racial difference affecting vascular lesions between Japanese and western populations. For Japanese in Japan, small intracerebral artery disease is more common than in persons of Japanese ancestry in Hawaii $(24,25)$. Inzitari et al also reported that orientals had intracranial arterial lesions more frequently than extracranial arterial lesions (26). The association between blood pressure and stroke in an eastern Asian population seemed stronger than in a western population, and 
the cholesterol concentration was a less important factor in the eastern Asian population (27). Therefore, our results may represent characteristics specific to Japanese and other orientals.

In this study, we examined other silent subcortical brain lesions on MRI. These lesions were also frequently observed in lacunar infarction. The EPS grade was higher in lacunar infarction than in the others. The Scheltens' scores of PVH, BGH, and total score were higher in the lacunar infarction group than in the others, and WMH score was higher in the lacunar infarction group than in the cardioembolic infarction group. Based on these results, we considered that the pathogenesis of these lesions was small-vessel vasculopathy being similar to that of lacunar infarction. EPS is closely related to arteriosclerosis of small penetrating arteries, aging, and hypertension (28). $\mathrm{PVH}$ is considered to be an ischemic lesion resulting from smallvessel disease $(29,30)$. Although the pathogenesis of white matter high intensity lesions on MRI has not been elucidated yet, such lesions may be caused by ischemic processes and seem to be associated with hypertension, aging $(8,31,32)$, and arteriosclerosis of small penetrating arteries (33). Thus, WMH could be associated primarily with arteriosclerosis of small vessels, but also partly with atherosclerosis of large vessels. A previous study also suggested that WMH is related to atherosclerosis of large vessels (34). We have previously suggested that the most important and common underlying mechanism for SSBI, focal white matter lesions, and PVH is hypertensive small-vessel vasculopathy (5). Thus, we considered that SSBI and other silent subcortical lesions were related to small-vessel disease.

Hypertension is the strongest risk factor for ischemic stroke, especially in lacunar infarction (20-22). We found that hypertension was frequent in lacunar infarction, but there was no statistically significant difference among the subtypes of ischemic stroke. Hypertension was the most potent risk factor for SSBI as demonstrated by multivariate logistic regression. Several studies have shown that hypertension is a significant risk factor for silent cerebral infarction $(3,5,6,10,12)$. We have also reported that $28 \%$ of asymptomatic adults with SSBI have hypertension, and hypertension is a significant risk factor for SSBI (5). Mast et al reported that the prevalence of hypertension is $73 \%$ in lacunar infarction and $64 \%$ in non-lacunar infarction (35). These results indicated that hypertension is a common risk factor for both SSBI and lacunar infarction. Millikan and Futrell reviewed several reports on lacunar infarction, and suggested that hypertension is not a specific risk factor, as the incidences of hypertension in patients with lacunes and large infarcts are similar (36). Hypertension may be an essential risk factor for lacunar infarction, as well as for other types of infarction.

\section{Conclusion}

In conclusion, the frequencies of SSBI and EPS were higher in lacunar infarction patients than in patients with other types of infarction. Other silent subcortical brain lesions, such as periventricular hyperintensity, white matter hyperintensity, and basal ganglia hyperintensity, were also more commonly observed in lacunar infarction patients than in patients with other types of infarction. The existence of SSBI and EPS in patients who developed symptomatic ischemic stroke suggests the involvement of small-vessel vasculopathy. Hypertension was an important risk factor for SSBI and other silent subcortical lesions, as well as for lacunar infarction.

Acknowledgements: This research was supported by research funds "Medical Economics in Cardiovascular Disease (1997-), and Clinical Significance in Progression of Cerebrovascular Lesions with Aging (1997-) from the Japanese Ministry of Health and Welfare, and by a research fund for Stroke Recurrence and Vascular Dementia (1997-)" from the Foundation of Shimane Institute of Health Science.

\section{References}

1) Kobayashi S, Okada K, Yamashita K. Incidence of silent lacunar lesion in normal adults and its relationship to cerebral blood flow and risk factors. Stroke 22: 1379-1383, 1991.

2) Matsubayashi S, Shimada K, Kawamoto A, Ozawa A. Incidental brain lesions on magnetic resonance imaging and neurobehavioral functions in the apparently healthy elderly. Stroke 23: 175-180, 1992.

3) Shimada K, Kawamoto A, Matsubayashi K, Ozawa T. Silent cerebrovascular disease in the elderly: Correlation with ambulatory pressure. Hypertension 16: 692-699, 1990.

4) Price TR, Manolio TA, Kronmal RA, et al. Silent brain infarction on magnetic resonance imaging and neurological abnormalities in community-dwelling older adults: the cardiovascular health study. Stroke 28: 1158-1164, 1997.

5) Kobayashi S, Okada K, Koide H, Bokura H, Yamaguchi S. Subcortical silent brain infarction as a risk factor for clinical stroke. Stroke 28: 19321939, 1997.

6) Shinkawa A, Ueda K, Kiyohara Y, et al. Silent cerebral infarction in a community-based series in Japan: the Hisayama study. Stroke 26: 380385, 1995.

7) Bryan RN, Wells SW, Miller TJ, et al. Infarctlike lesions in the brain: prevalence and anatomic characteristics at MR imaging of the elderlydata from the cardiovascular health study. Radiology 202: 47-54, 1997.

8) Ylikoski A, Erkinjuntti T, Raininko R, Sarna S, Sulkava R, Tilvis R. White matter hyperintensities on MRI in the neurologically nondiseased elderly: analysis of cohorts of consecutive subjects aged 55 to 85 years living at home. Stroke 26: 1171-1177, 1995.

9) Kase CS, Wolf PA, Chodosh EH, et al. Prevalence of silent stroke in patients presenting with initial stroke: the Framingham study. Stroke 20: 850-852, 1989.

10) Boon A, Lodder J, Raak LH, et al. Silent cerebral infarcts in 755 consecutive patients with first-ever supratentorial ischemic stroke: relationship with index-stroke subtype, vascular risk factors, and mortality. Stroke 25: 2384-2390, 1994.

11) Chamorro A, Saiz A, Vila N, et al. Contribution of arterial blood pressure to the clinical expression of lacunar infarction. Stroke 27: 388-392, 1996.

12) Okada Y, Sadoshima S, So Y, Ishizuka T, Fujishima M. Asymptomatic cerebrovascular lesions in hypertensive cerebral hemorrhage. Jpn J stroke 27: 187-191, 1992.

13) National Institute on Neurological Disorders and Stroke Ad Hoc Committee: Classification of cerebrovascular diseases III. Stroke 21: 637-676, 1990.

14) Sawada T, Oita J, Okamoto K, et al. Definition and diagnostic criteria of asymptomatic cerebrovascular diseases. Jpn J Stroke 19: 489-493, 1997.

15) Bokura H, Kobayashi S, Yamaguchi S. Distinguishing silent lacunar infarction from enlarged Virchow-Robin spaces; a magnetic resonance imaging and pathological study. J Neurol 245: 116-122, 1998.

16) Scheltens $P$, Barkhof $F$, Leys $D$, et al. A semiquantative rating scale for the assessment of signal hyperintensities on magnetic resonance imag- 


\section{ADACHI et al}

ing. J Neurol Sci 114: 7-12, 1993.

17) Rothwell PM, Gibson RJS, Slattery J, Warlow CP. The equivalence of measurements of carotid stenosis: a comparison of three methods on 1001 angiogram. Stroke 25: 2435-2439, 1994.

18) Korogi $Y$, Takahashi M, Mabuchi N, et al. Intracranial vascular stenosis and occlusion: diagnostic accuracy of three-dimensional, Fourier transform, time-of-flight MR angiography. Radiology 193: 187-193, 1994.

19) Fujita N, Hirabuki N, Fujii K, et al. MR imaging of middle cerebral artery stenosis and occlusion: value of MR angiography. AJNR 15: 335341, 1994.

20) Fisher CM. Lacunes: small, deep cerebral infarcts. Neurology 15: 774 784, 1965.

21) Fisher CM. Lacunar stroke and infarcts: a review. Neurology 32: 871876, 1982.

22) You R, McNeil JJ, O'Malley HM, Davis SM, Donnan GA. Risk factors for lacunar infarction syndromes. Neurology 45: 1483-1487, 1995.

23) Laloux P, Ossemann M, Jamart J. Stroke subtypes and risk factors associated with silent infarctions in patients with first-ever ischemic stroke or transient ischemic attack. Acta Neurol Belg 94: 17-23, 1994.

24) Reed D, Jacobs DR, Hayashi T, et al. A comparison of lesions in small intracerebral arteries among Japanese men in Hawaii and Japan. Stroke 25: 60-65, 1994

25) Mitsuyama Y, Thompson LR, Hayashi T, et al. Autopsy study of cerebrovascular disease in Japanese men who lived in Hiroshima, Japan, and Honolulu, Hawaii. Stroke 10: 389-395, 1979.

26) Inzitari D, Hachinski VC, Taylor DW, Barnett HJM. Racial differences in the anterior circulation in cerebrovascular disease: How much can be explained by risk factors? Arch Neurol 47: 1080-1084, 1990.

27) Eastern stroke and coronary heart disease collaborative research group. Blood pressure, cholesterol, and stroke in eastern Asia. Lancet 352: 1801-
$1807,1998$.

28) Braffman BH, Zimmerman RA, Trojanowski JQ, Gonatas NK, Hickey WF, Schlaepfer WM. Brain MR: pathologic correlation with gross and histopathalogy. 1. lacunar infarction and Virchow- Robin spaces. Radiology 151: 551-558, 1988.

29) Streifler JY, Eliasziw M, Benavente OR, Hachinski VC, Fox AJ, Barnett HJM, for the North American Symptomatic Carotid Endarterectomy Trial. Lack of relationship between leukoaraiosis and carotid artery disease. Arch. Neurol 52: 21-24, 1995.

30) Adachi T, Takagi M, Hoshino H, Inafuku T. Effect of extracranial carotid artery stenosis and other risk factors for stroke on periventricular hyperintensity. Stroke 28: 2174-2179, 1997.

31) Schmidt R, Fazekas F, Kleinert G, et al. Magnetic resonance imaging signal hyperintensities in the deep and subcortical white matter: a comparative study between stroke patients and normal volunteers. Arch Neurol 49: 825-827, 1992.

32) Jørgensen HS, Nakayama H, Raaschou HO, Olsen TS. Leukoaraiosis in stroke patients: The Copenhagen stroke study. Stroke 26: 588-592, 1995.

33) Goto K, Ishii N, Fukasawa H. Diffuse white-matter disease in the geriatric population: a clinical, neuropathological, and CT study. Radiology 141: 687-695, 1981.

34) Isaka $Y$, Nagano $K$, Narita $M$, Ashida $K$, Imaizumi M. High signal intensity on T2-weighted magnetic resonance imaging and cerebral hemodynamic reserve in carotid occlusive disease. Stroke 28: 354-357, 1997.

35) Mast H, Thompson JLP, Lee S, Mohr JP, Sacco RL. Hypertension and diabetes mellitus as determinant of multiple lacunar infarcts. Stroke 26: 30-33, 1995.

36) Millikan C, Futrell N. The fallacy of the lacune hypothesis. Stroke 21: 1251-1257, 1990. 